\title{
A SYNOPSIS OF THE CHRYSIDID GENERA OF NEOTROPICAL AMERICA (CHRYSIDOIDEA, HYMENOPTERA)*
}

\author{
By LyNN S. Kimsey AND RichaRd M. BohaRT \\ Department of Entomology, University of California, Davis 95616
}

The chrysidid fauna of Central and South America, including Mexico, differs considerably from that of North America. There are only a few species whose range extends into both regions. The genera Cleptes, Mesitiopterus, Microsega, Muesebeckidium, Pseudolopyga, Minymischa, Xerochrum, Microchridium, Hedychreides, Chrysura, and Chrysurissa are not reported from South or Central America. A number of genera are not found north of Costa Rica, including Duckeia, Nesogyne and Gaullea. Trichrysis and Neochrysis have undergone extensive species radiation in the neotropics. The few species in these two genera occurring in North America are their northernmost representatives.

The faunas of Baja California, north central and northwestern Mexico seem to be essentially temperate in origin. The genera Parnopes, Spintharosoma and Ceratochrysis belong to this temperate fauna and are considered in this survey because they are found in Mexico. Several of the genera found only in the southwestern United States may be present in Baja California, Sonora and Chihuahua, Mexico, so specimens from this region should probably be run through the key to the North American genera (Bohart and Kimsey, 1980). The chrysidid fauna of the Caribbean islands, excluding Trinidad and Tobago, appears to be primarily temperate in origin, with only one endemic genus, Nesogyne.

The principal work on neotropical Chrysididae has been done by Juan Brèthes, August Brullé, Robert du Buysson, Peter Cameron, Alphonse Ducke, Felix Guérin-Méneville, Karl Krombein, Alexandro Mocsáry and Maximilien Spinola. Lesser work has been done by R. Benoist, H. Bischoff, R. M. Bohart, E. T. Cresson, A. da Costa Lima, Andrea Dahlbom, Vicente Perez-D'Angello, Johann Fabricius, Claude Joseph (H. Janvier), L. S. Kimsey, Walter Linsenmaier and Flaminio Ruiz.

*Manuscript received by the editor August 26, 1980. 
The two largest chrysidid genera in the neotropics are Trichrysis and Neochrysis, whose dominance roughly corresponds to Ceratochrysis and Chrysis in the nearctic fauna. Detailed examination of Trichrysis in both hemispheres has revealed four distinct groups. Old World Trichrysis can be divided into two subgenera, based on whether the discoidal cell is complete (Trichrysis s.s.) or incomplete (Chrysidea). The New World Trichrysis can also be divided into two groups based on the same characteristic. However, New World Trichrysis are different from the Old World forms since they have a distinct and almost articulated telomere on the male genital capsule (fig. 24). This discrete telomere has not been found in Old World Trichrysis (fig. 22).

We therefore propose the establishment of two new subgenera in the genus Trichrysis. An asterisk after a species name indicates that we have not seen the type.

\section{Trichrysis Lichtenstein}

Trichrysis (Caenochrysis) Kimsey and Bohart, new subgenus (derivation: Kaeno = new, Gr.)

Subgenerotype: Chrysis tridens Lepeletier

Included species: amazonica Mocsáry, azteca Mocsáry, brasiliana

Guérin, carinata Say*, divergens Cresson, gibbosa Mocsáry, mathani Ducke*, mucronata Brullé*, nigropolita Bischoff, parvula Fabricius, taschenbergi Mocsáry, triangulifera Mocsáry, and tridens Lepeletier*.

Discussion: Members of the subgenus can be recognized by the sometimes thin but completely sclerotized outer veins of the discoidal cell in the forewing (fig. 18), and the presence of a telomere on the paramere (fig. 24).

Trichrysis (Lorochrysis) Kimsey and Bohart, new subgenus (derivation: Loron = strap, Gr.)

Subgenerotype: Chrysis doriae Gribodo

Included species: areolata Mocsáry, armata Mocsáry, boscii Buysson, crotonis Buysson, doriae Gribodo, puberula Spinola, quadririmosa Mocsáry, saussurei Mocsáry.

Discussion: This subgenus is distinguished by a faint or incompletely sclerotized discoidal cell in the forewing, with at least the anterior vein faint or lacking (fig. 17) and the presence of a telomere on the paramere. In general the species are smaller than those of Caenochrysis. 
Trichrysis (Trichrysis) Lichtenstein

Subgenerotype: Sphex cyanea Linnaeus

Discussion: Trichrysis s.s. is an eastern hemisphere group, recognizable by the completely sclerotized discoidal cell and no telomere on the paramere (fig. 22).

\section{Trichrysis (Chrysidea) Bischoff}

Subgenerotype: Chrysis pumila Klug

Discussion: Chrysidea is also an eastern hemisphere group which lacks a telomere and has the forewing discoidal cell incomplete, since at least the anterior vein of the discoidal cell is lacking or faintly indicated.

\section{Neochrysis Linsenmaier}

Neochrysis is the largest genus in the neotropics. This genus was divided into four subgenera by Bohart (1966). We have further elaborated the characteristics of each subgenus and separated the neotropical species accordingly. The list of species under each subgenus may not be complete. Each list is based on species where we have seen the type or seen reliably determined material (type not seen indicated by an asterisk).

Neochrysis Linsenmaier, 1959. Generotype by original designation, Chrysis punctatissima Spinola, 1840 (nec Villers, 1789) $=$ Chrysis carina Brullé, 1846.

T-III pit row obsolescent, pits, when discernible, no larger than other punctures (fig. 11); 4 distal teeth on T-III; frons without a cross carina or rarely a few traces of it, no strong carina leading back from brow to partly enclose midocellus; pronotum without a complete lateral carina; propodeum without a strong median projection just behind metanotum.

Partial list of included species: cameroni (Buysson), carina Brullé, deuteroleuca (Mocsáry), inseriata (Mocsáry), lecointei Ducke, montezuma (Cameron), paraensis (Ducke).

Pleurochrysis Bohart, 1963, new name for Pleurocera Guérin (nec Rafinesque, 1818). Subgenerotype by monotypy, Chrysis viridis Guerin, 1842 (nec Oliver, 1790) = Chrysis bruchi Brèthes, 1902.

T-III pit row usually well developed and forming a strong groove, pits larger than punctures, at least laterally (fig. 12); 4 distal teeth on T-III or rarely 6; frons with or without a cross carina (fig. 13), 
sometimes a strong carina leading back to partly enclose midocellus; pronotum without a complete lateral carina; propodeum without a projection behind metanotum.

Partial list of included species: acúta (Brèthes), alfkeni (Ducke), ameginoi (Brèthes), ancilla (Buysson), bruchi (Brèthes), cavifrons (Brullé), charruana (Brèthes), chilicola (Mocsáry), fasciifera (Bischoff), lynchi (Brèthes), mendozana (Mocsáry), missionera (Brèthes), postica (Brullé), ypirangensis (Buysson).

Exochrysis Bohart, 1963, Subgenerotype by original designation, Chrysis panamensis Cameron, 1888.

T-III pit row or groove well developed; 4 distal teeth on T-III; frons with a cross carina which branches back to enclose or partly enclose midocellus; pronotum with at most a partial lateral carina (fig. 5); propodeum with a strong median projection just behind metanotum (fig. 5). Partial list of included species: imperforata (Gribodo), leucophris (Mocsáry), leucostigma (Mocsáry), panamensis (Cameron), spinigera (Spinola).

Ipsiura Linsenmaier, 1959. Subgenerotype by original designation, Chrysis marginalis Brullé.

T-III pit row or groove well developed; $2-6$ distal teeth on T-III; frons sometimes with a cross carina but always with a carina leading back from brow to partly enclose midocellus; pronotum with a sharp and practically complete lateral carina or flange (fig. 4); propodeum without a projection behind metanotum (fig. 4).

Partial list of included species: albibasalis (Mocsáry), bisulcata (Ducke), brevispinosa (Ducke), ellampoides (Ducke), friesiana (Ducke), genbergi (Dahlbom)*, klugi Dahlbom, leucobasis (Mocsáry), leucocheila (Mocsáry), leucochiloides (Ducke), longiventris (Ducke), marginalis (Brullé), myops (Buysson), neolateralis Bohart, obidensis (Ducke), pilifrons (Cameron).

Argochrysis Kimsey and Bohart, new genus (derivation: Argos = white Gr.)

Generotype: Chrysis mesillae Cockerell

Included species: mesillae (Cockerell), trochilus (Buysson) and a number of undescribed species.

Discussion: This genus is distinguished by $\mathrm{F}-\mathrm{I}$ as long or longer than F-II in both sexes; tongue short, less than half as long as flagellum from basal fold to apex; mesopleuron with shallow transverse groove 
or no groove; propodeal teeth tapering and apically pointed (fig. 14); (fig. 7) leg joints, and apical rim of T-III often white or hyaline; paramere digitate and or lobate (fig. 19); cuspis broadly rounded, and digitus equal to or shorter than cuspis and apically rounded (fig. 19).

Examination of the type species of Spintharosoma Zimmermann, Chrysis chrysonota Dahlbom, from the Ethiopian Region reveals that the American species previously placed in this genus are not congeneric.In $S$. chrysonota the tongue is long, the propodeal teeth clublike (fig. 15), paramere simple and rounded and cuspis slender and tapering (fig. 21), the male F-I is less than one third as long as FII, and the mesopleuron divided into two parts by a deep transverse groove. American species also differ from Spintharina (generotype Chrysis vagans Radovskowsky). Spintharina has two large teeth on the mesopleuron, lobate propodeal teeth (fig. 16), and male genitalia with simple unlobate paramere and long slender digitus and cuspis (fig. 20).

\section{Gaullea Buysson}

The genus Gaullea is an obscure and poorly known genus. Gaullea was originally described from a single female, collected in Chaco, Santiago del Estero, Argentina. We have seen an additional 2 females and 3 males from Santiago del Estero, Argentina, plus the type. This genus is characterized by the very short RS stub, lack of a discoidal cell in the forewing and denticulate rim of T-III (figs. 6, 10). Linsenmaier (1959) placed Gaullea near Euchroeus. However, after close examination of the wing venation we have placed Gaullea near Argochrysis, because a crease in the wing indicates that the marginal cell would be closed if the RS stub were extended. The closed marginal cell is characteristic of Chrysis and its relatives. The expanded and bladelike metanotum and denticulate rim of T-III suggests that Gaullea is closely related to Argochrysis. The male genitalia of Gaullea is similar to that of Chrysis, with a simple though somewhat apically lobate paramere, broad foliaceous digitus and slender setose cuspis (fig. 23).

\section{BIOLOGY}

Host records for the neotropical Chrysididae are incomplete at best and nonexistant for Nesogyne, Cleptidea, Amisega, Pseudospinolia and Gaullea. The Amiseginae are typically phasmatid egg parasites. The remaining genera are wasp and bee parasites (table 1). 


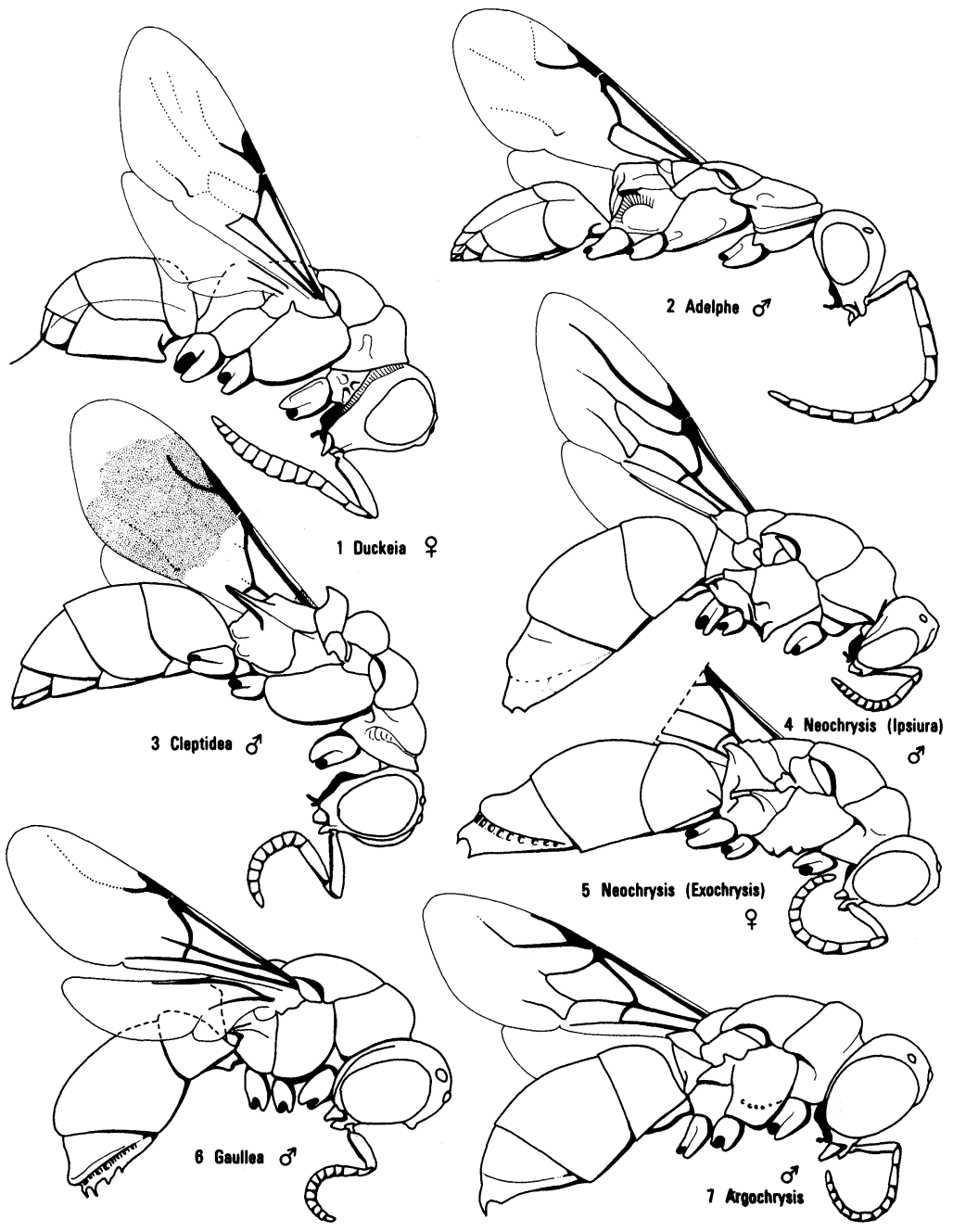

Figs. 1-7, Lateral view of body. 1. Duckeia cyanea Costa Lima. 2. Adelphe mexicana Mocsáry. 3. Cleptidea aurora (Smith). 4. Neochrysis (Ipsiura) marginalis (Brullé). 5. Neochrysis (Exochrysis) panamensis (Cameron). 6. Gaullea argentina Buysson. 7. Argochrysis mesillae (Cockerell). 


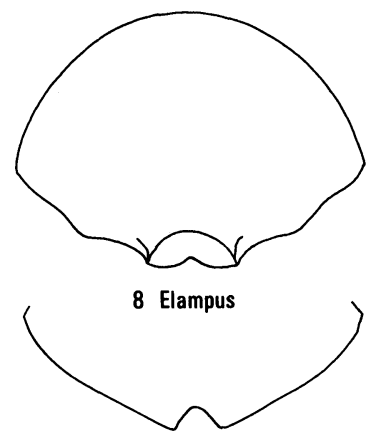

9 Omalus

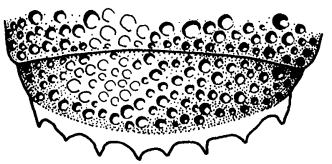

10 Gaullea
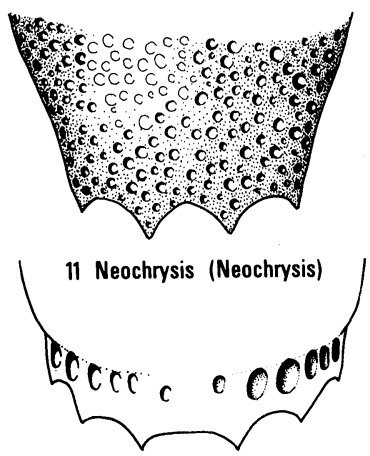

12 Neochrysis (Pleurochrysis)
13 Neochrysis (Pleurochrysis)
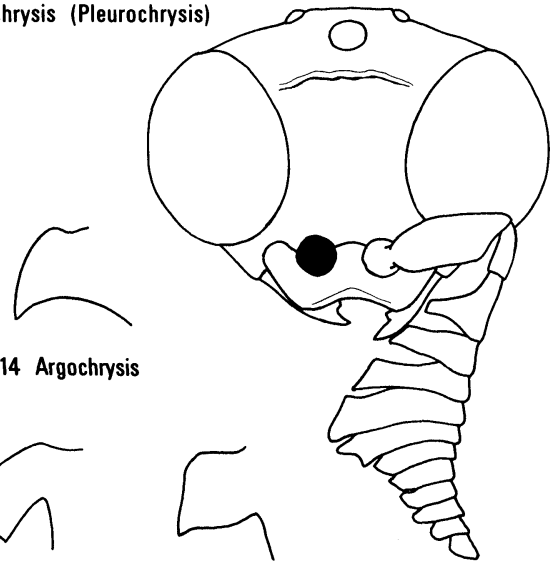

15 Spintharosoma

16 Spintharina

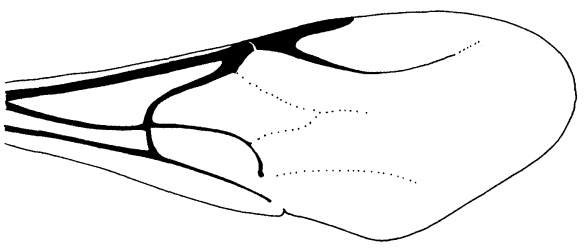

17 Trichrysis (Lorochrysis)

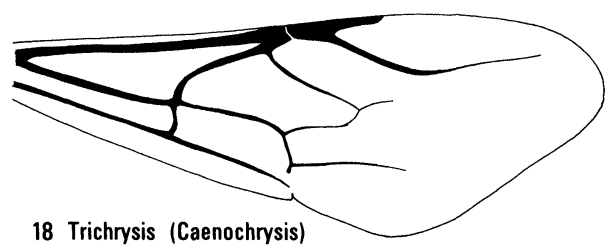

Figs. 8-12, Apical margin of tergum III. 8. Elampus nitidus (Aaron). 9. Omalus butleri Bohart and Campos. 10. Gaullea argentina Buysson. 11. Neochrysis (Neochrysis) carina (Brullé). 12. Neochrysis (Pleurochrysis) bruchi (Brèthes). Fig. 13, Front view of male face of Neochrysis (Pleurochrysis) bruchi (Brèthes). Figs. 14-16, Anteroventral view of propodeal tooth. 14. Argochrysis mesillae (Cockerell). 15. Spintharosoma chrysonota (Dahlbom). 16. Spintharina vagans Radovskowsky. Figs. 17-18, Forewing venation. 17. Trichrysis (Lorochrysis) doriae Gribodo. 18. Trichrysis (Caenochrysis) tridens (Lepeletier). 


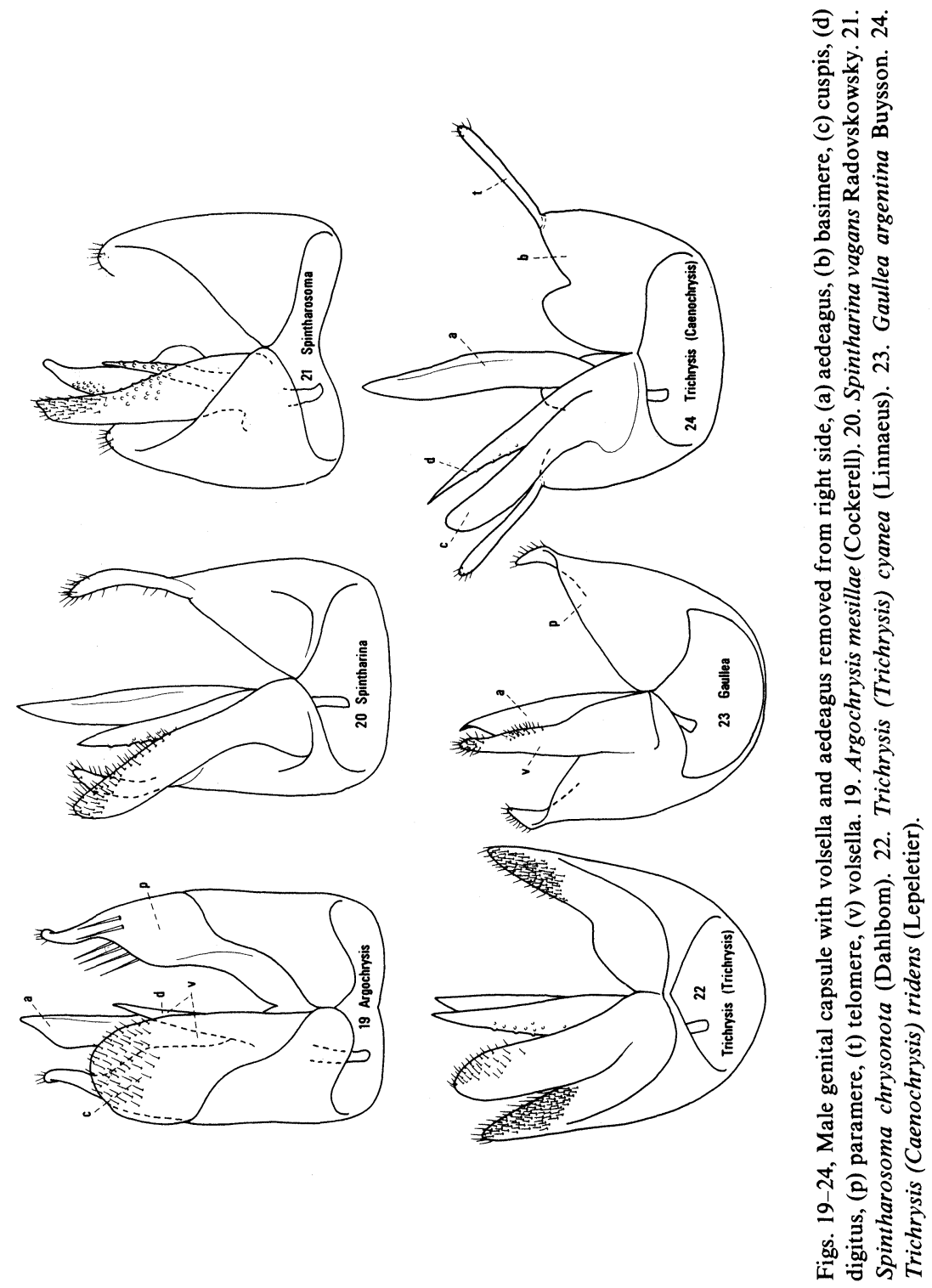


Very little biological work has been done on the neotropical chrysidids. Host data has been reported for Omalus by Benoist (1942), Trichrysis, Chrysis and Neochrysis by Perez-D'Angello (1968), Coville and Coville (1980), Ducke (1904), Fritz (pers. comm), Janvier (1933) and Ruiz (1938), Adelphe and Mesitiopterus by Krombein (1957) and Duckeia by Costa Lima (1936).

Table I. Host records, distribution and number of species of neotropical Chrysididae.

\section{Subfamily Cleptinae}

Cleptidea-host not recorded; southern Mexico to Brazil; 6 species.

Subfamily Amiseginae

Duckeia-Phasmatidae: Prisopus sp. (eggs); Brazil; 1 species. Amisega-Phasmatidae?: Costa Rica to Brazil; 4 species. Mesitiopterus - Phasmatidae: Diapheromera sp. (eggs); southern Mexico to Venezuela; 2 species.

Adelphe - Phasmatidae: Anisomorpha; Mexico to Brazil; 4 species. Nesogyne - host not recorded; Dominican Republic; 1 species

\section{Subfamily Elampinae}

Omalus-Sphecidae: Pemphredoninae: Stigmus; Mexico, Brazil, Argentina and Chile; 5 species.

Elampus - Sphecidae (not recorded in neotropics); Cuba, Venezuela, Brazil, Argentina; Chile; 3 species.

Holopyga-Sphecidae (not recorded in neotropics); Dominican Republic, Mexico, Brazil, Peru, Argentina, Chile; 7 species.

Hedychridium-Sphecidae and Apoidea (not recorded in neotropics); Mexico to Chile; 3 species.

Hedychrum -Sphecidae: Philanthinae? (not recorded in neotropics); widespread; 6 species.

\section{Subfamily Chrysididinae}

Chrysis-Eumenidae: Hypodynerus and Stenodynerus; widespread; 12 species.

Ceratochrysis-Eumenidae, Sphecidae; Mexico: Chihuahua and Baja California; 2 species. 
Pseudospinolia-host not recorded in neotropics; Chile; 1 species. Argochrysis-Sphecidae: Ammophila (not recorded in neotropics); Mexico: Chihuahua and Baja California. 1 species.

Gaullea-host not recorded; Argentina; 1 species.

Trichrysis-Sphecidae: Trypoxylon, Eumenidae; widespread; 20 species.

Neochrysis-Sphecidae: Podium, Trypoxylon, Sceliphron; widespread; 32 species.

\section{Subfamily Parnopinae}

Parnopes-Sphecidae: Bembicini (prob. only on Microbembex in Mexico), northern Mexico, Sonora; 1 species.

\section{ACKNOWLEDGMENTS}

We are very grateful to Ignacio and Raquel Cid del Prado for their work on translating the generic key into Spanish.

Key to the Genera of Neotropical Chrysididae For brevity in the key the following abbreviations have been used: $\mathrm{T}$ for tergum, $\mathrm{S}$ for sternum and $\mathrm{RS}$ for radial sector.

1. Abdominal venter convex $\ldots \ldots \ldots \ldots \ldots \ldots \ldots \ldots . \ldots . \ldots . \ldots 2$ Abdominal venter flat to concave ................ 7

2. T-I-II together dorsally subequal to or shorter than length of T-III-IV (fig. 3); pronotum with submedial transverse groove (fig. 3); upper frons with groove descending from midocellus (Cleptinae) .................. Cleptidea Mocsáry T-I-II together dorsally much longer than length of T-III-IV (figs $1,2)$; pronotum usually without transverse groove; upper frons without median groove (Amiseginae) ......... 3

3. Propodeum rounded posterolaterally (fig. 1), pronotal lobes usually not reaching tegulae (fig. 1) $\ldots \ldots \ldots \ldots \ldots \ldots 4$

Propodeum toothed posterolaterally (fig. 2), pronotal lobes reaching tegulae $\ldots \ldots \ldots \ldots \ldots \ldots \ldots \ldots \ldots$

4. Pronotum medially depressed or grooved; S-II produced, keeled anteriorly; gena flanged; propodeum strongly produced, posterior face almost flat (fig. 1)... . Duckeia Costa Lima Pronotum not medially depressed, S-II simple; gena not flanged; propodeum broadly rounded posteriorly .......... 5 
5. Scutum with notauli $\ldots . \ldots \ldots \ldots \ldots$ Amisega Cameron Scutum without notauli ......... Mesitiopterus Ashmead

6. Pronotum with transverse anterior groove, female with normal wings; propodeal tooth broad, subtriangular (fig. 2) ...... .......................... Adelphe Moscáry

Pronotum without transverse carina, female brachypterous, propodeal tooth slender and digitate

Nesogyne Krombein

7. RS stub sclerotized for less than half marginal cell length (if vein is extended by its traces and wing crease to wing margin); outer veins of discoidal cell faint or barely indicated (fig. 6); tarsal claws various ......................... 8

RS stub sclerotized for more than half marginal cell length (if vein is extended by its traces and wing crease to wing margin); outer veins of discoidal cell usually sclerotized (figs. 4, 5, 7); claws simple ........................ 14

8. Last visible tergum spiculate along apical margin; claws simple

Last visible tergum not spiculate along apical margin; claws dentate (Elampinae) ..................... 10

9. Tongue exserted and reaching near base of abdomen, last visible tergum subapically depressed on either side of midline; females with three normally visible terga, males with four (Parnopinae) ................. Parnopes Latreille

Tongue short, not exserted; last visible tergum not subapically depressed; both sexes with three normally visible terga (Chrysidinae) .................. Gaullea Buysson

10. Hindtarsal claw long and slender, with a medial, nearly perpendicular tooth; frons with close punctation along inner eye margins (punctures less than one puncture diameter apart; scutellum not anteriorly carinate $\ldots \ldots \ldots \ldots \ldots \ldots \ldots$. Hedychridium Abeille de Perrin

Hindtarsal claw without a single, medial, nearly perpendicular tooth; other characteristics various $\ldots \ldots \ldots \ldots \ldots \ldots 11$

11. Medial vein essentially straight or weakly curved; hindtibia and usually midtibia with a definite pit on inner surface toward apex; female S-III usually with a nodular apicomedial projection Hedychrum Latreille Medial vein strongly arched; hindtibia and midtibia without a pit on inner surface; female S-III without an apicomedial projection ................................ 12 
12. Medial cell with numerous dark microsetae; last tergum with punctate medially unnotched apical margin; face hollowed and crossridged; hindtarsal claws usually with two to five subsidiary teeth (rarely only one subsidiary) ........... Holopyga Dahlbom

Medial cell essentially asetose; last tergum apicomedially impunctate and usually notched or compressed and snoutlike (figs. 8, 9); face hollowed without crossridging; hindtarsal claws various ........................ 13

13. T-III apicomedially notched (fig. 9), emarginate or simple not compressed and snoutlike; metanotum rounded, not conical or produced and bladelike; scutum impunctate or with punctures clustered along or between notauli, except chilensis (Mocsáry) ..................... Omalus Panzer

T-III apicomedially compressed and snoutlike (fig. 8); metanotum conical or produced and bladelike; scutum densely punctate .................... Elampus Spinola

14. Radial sector ending before wing margin so that marginal cell is broadly open (fig. 4) $\ldots \ldots \ldots \ldots \ldots \ldots \ldots \ldots \ldots$

Radial sector and its faintly sclerotized remnant narrowly closing marginal cell or almost so (figs. 7, 17, 18) .... 19

15. T-III apical margin smooth, slightly prolonged medially, especially in male, anterolateral angles of T-I not unusually sharp .................... Pseudospinolia Linsenmaier

T-III ending in four to six teeth; anterolateral angles of T-I unusually sharp $\ldots \ldots \ldots \ldots \ldots \ldots \ldots \ldots \ldots . \ldots \ldots$

16. Propodeum with strong median projection just behind metanotum (fig. 5) .......... Neochrysis (Exochrysis) Bohart Propodeum without strong median projection just behind metanotum ........................ 17

17. Pronotum with sharp and practically complete lateral carina or flange (fig. 4) T-III pit row or groove well developed (as in fig. 12) ............. Neochrysis (Ipsiura) Linsenmaier

Pronotum without a sharp or practically complete lateral carina or flange; T-III pit row various .............. 18

18. T-III pit row obsolescent, pits, when discernible, no larger than other punctures (fig. 11); frons without cross carina, rarely with traces remaining and no carinae extending back toward ocelli ................ Neochrysis s.s. Linsenmaier T-III pit row well developed and forming a strong groove, at least 
some pits larger than punctures (fig. 12); frons usually with cross carina and carinae extending back toward ocelli .... ................. Neochrysis (Pleurochrysis) Bohart

19. Outer veins of discoidal cell faint (fig. 17) .............. ........... Trichrysis (Lorochrysis) Kimsey and Bohart

Outer veins of discoidal cell sclerotized (fig. 18) ....... 20

20. T-III not apicomedially notched, usually with three to six teeth ............................... 21

T-III apicomedially deeply notched, lacking subsidiary teeth, except on either side of notch, central Mexico north ... 22

21. T-III ending in three or fewer teeth or angles ............. ......... Trichrysis (Caenochrysis) Kimsey and Bohart T-III ending in four or six teeth or angles

Chrysis Linnaeus

22. Tibiae with pale basal markings and T-III rim whitish; or metanotum conically produced (fig. 7)

Argochrysis Kimsey and Bohart

Tibiae without pale markings and T-III rim not whitish; metanotum not produced...$\ldots \ldots \ldots$..... Ceratochrysis Cooper

Clave para les géneros neotropicales de la familia Chrysididae

1. Región ventral del abdomen convexa $\ldots \ldots \ldots \ldots \ldots . \ldots 2$

Región ventral del abdomen plana o cóncava ........ 7

2. T-I-II dorsalmente subiguales o más cortos que el largo de T-III-

IV (fig. 3); pronotum con hendidura submedia transversa (fig. 3); frente superior con una hendidura descendiendo del ocelo medio (Cleptinae) .......... Cleptidea Mocsáry

T-I-II dorsalmente tan largos que el largo de T-III-IV (figs 1, 2); pronotum sin o con hendidura transversal; frente superior sin hendidura media (Amiseginae) ................ 3

3. Propodeum redondeado posteriolateralmente (fig. 1), lóbulos pronotales usualmente sin alcanzar la tégula (fig. 1) ...4 4

Propodeum dentado posteriolateralmente (Fig. 2), lóbulos pronotales alcanzando la tégula $\ldots \ldots \ldots \ldots \ldots \ldots 6$ 
4. Pronotum en su parte media hundido o con hendidura; S-II forma una projeción angular anteriormente; gena con projeción o borde; proprodeum evidentemente pronunciado posteriormente, extremo posterior casi plano (fig. 1) ...... ...................... Duckeia Costa Lima

Pronotum sin depresión media; S-II simple; gena sin projeción alar; propodeum distintamente redondeado posteriormente ............................... 5

5. Scutum con notauli ...............Amisega Cameron Scutum sin notauli ............ Mesitiopterus Ashmead

6. Pronotum con hendidura transversal; hembra con alas normales; diente propodeal ancho, subtriangular (fig. 2)

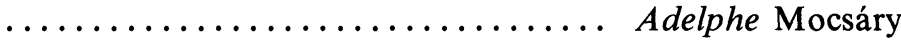

Pronotum sin hendidura transversal; hembra con alas brachypterous; diente propodeal alargado y angosto ........ ....................... Nesogyne Krombein

7. RS fragmento esclerotizado, menor que la mitad del largo de la célula marginal (si la vena es extendida por sus trazos y plieque del ala marginal); venas externas de la célula discoidal ténues o apenas visibles (fig. 6); uñas tarsales variables . 8

RS escleratizado más de la mitad del largo de la célula marginal (si la vena es extendida por sus trazas y plieque de ala marginal); venas externas de la célula discoidal usualmente escleratizado (figs $4,5,7$ ); uñas tarsales simples ..... 14

8. Último tergum visible, espiculado a lo largo del margin apical; uńas tarsales simples $\ldots \ldots \ldots \ldots \ldots \ldots \ldots \ldots$

Último tergum visible, no espiculado a lo largo del margin apical; uñas tarsales dentadas (Elampinae) ........ 10

9. Lengua extendida, alcanza cerca la base del abdomen; último tergum visible, subapicalmente hundido en su parte media; hembras con tres normalmente visibles terga, machos con cuatro (Parnopinae) ............ Parnopes Latreille

Lengua corta, no se extiende; último tergo visible no subapicalmente hundido; ambos sexos con tres normalmente visibles terga (Chrysididinae) ............. Gaullea Buysson

10. Uñas de les tarsos posteriores largas y delgadas, con un diente medio perpendicular; frente con cerrada puntación a lo largo del margin interno del ojo (puntación menos que el diametro de una puntación distantes); scutellum no anteriormente con saliente Hedychridium Abeille de Perrin 
Uñas tarsales posteriores simples, diente medio perpendicular presente; otras características varias ............. 11

11. Vena media esencialmente recta o débilmente curvada; tibia posterior y tibia media usualmente con un claro hoyo en su superficie interna, cerca de su ápice; S-III de la hembra usualmente con una proyección nodular medioapical .....

Hedychrum Latreille

Vena media fuertemente arqueada; tibia posterior y media hoyo sobre su superficie interna; S-III de la hembra sin una proyección medioapical ................... 12

12. Célula media con numerosas microsetas obscuras; último tergo con mediana puntacion, no alcanza el margen apical; cara cóncava y con estriaciones transversales; unas traseras usualmente con 2 a 5 dientes subsidiarios (raremente uno) ..... Holopyga Dahlbom

Célula media esencialmente sin setas; último tergo sin mediana puntacion apical, usualmente con muescas o comprimido similar a una proyección truncada (figs 8,9 ); cara cóncava sin estriaciones transversales; uñas traseras varias ...... 13

13. T-III de la hembra medio apical con muescas (fig. 9), emarginado o simple no comprimido o comprimido y sin proyección truncada; metanotumo redondeado, no cónico o aplanado; scutum con puntación cerrada a lo largo o entre notauli, excepto chilensis (Mocsáry) .......... Omalus Panzer

T-III de la hembra medio apical comprimido y con projection truncada (fig. 8); metanotum con proyección cónica o aplana$\mathrm{da}$; scutum densamente punteado ...... Elampus Spinola

14. Sector radial termina antes del margen del ala, célula marginal es ampliamente abierta (fig. 4) ................. 15

Sector radial con célula marginal angosta y debilmente escleratizado o casi cerrada (figs. $7,17,18$ ) $\ldots \ldots \ldots \ldots . .19$

15. T-III margen apical liso, ligeramente prolongado, en su parte media, especialmente en machos; ángulos anterolateral de T-I no extraordinariamente agudos

Pseudospinolia Linsenmaier

T-III termina en cuatro o seis dientes; ángulos anterolaterales de T-I extraordinariamente agudo $\ldots \ldots \ldots \ldots \ldots \ldots 16$

16. Propodeum con fuerte proyección media justo atras del metanotum (fig. 5) .......... Neochrysis (Exochrysis) Bohart 
Propodeum sin fuerte proyección media, justo atras del metanotum .............................. 17

17. Pronotum con borde agudo y prácticamente completa carina lateral (fig. 4); T-III con hilera de hoyos bien desarrollados formando una hendidura (como fig. 12) ............. ................. Neochrysis (Ipsiura) Linsenmaier

Pronotum sin borde agudo o completa carina; T-III hilera de hoyos varios $\ldots \ldots \ldots \ldots \ldots \ldots \ldots \ldots \ldots \ldots . . \ldots \ldots$

18. T-III hilera de hoyos casi ausentes, hoyos cuando son discernibles, no tan largos que otras puntaciones (fig. 11); frente sin carina cruzada, raramente con remanentes y sin extenciones de la carinae hacia los ocelos .....................

Neochrysis s. s. Linsenmaier

T-III con hilera de hayos bien desarollado y formando un fuerte surco, algunos hoyos tan grandes que una puntacion (fig. 12); frente usualmente con carina cruzada y carinae extendiendase hacia los ocelos ....... Neochrysis (Pleurochrysis) Bohart

19. Venas externas de la célula discoidal débiles (fig. 17) ...... ........... Trichrysis (Lorochrysis) Kimsey and Bohart Venas externas de la célula discoidal esclerotizadas (fig. 18) ..

20. T-III medio apical sin muescas, usualmente con tres, cuarto o seis dientes $\ldots \ldots \ldots \ldots \ldots \ldots \ldots \ldots \ldots \ldots \ldots . \ldots 21$

T-III medio apical profundamente con muescas, dientes subsidarios ausentes, excepto sobre ambos lados de la muesca; central y norte de México ................... 22

21. T-III terminado en tres o algunos dientes o ángulos ........ ......... Trichrysis (Caenochrysis) Kimsey and Bohart T-III terminado en cuatro o seis dientes o ángulos 


\section{REFERENCES}

BENOIST, R.

1942. Les hymenopteres qui habitent les tiges de sonce aux environs de Quito. Ann. Ent. Soc. France III:75-90.

BOHART, R. M.

1966. The genus Neochrysis in America north of Mexico. Bull. Brooklyn Ent. Soc. 63:139-144.

Costa Lima, A DA.

1936. Sur un nouveau chryside: Duckeia Jubilaire E. L. Bouvier, pp. 173-175. Coville, R. E. and P. L. Coville.

1980. Nesting biology and behavior of Trypoxylon (Trypargilum) tenoctitlan in Costa Rica. Ann. Ent. Soc. Amer. 73:110-119.

JANVIER, $\mathrm{H}$.

1933. Etude biologique de quelques Hymenopteres du Chili. Ann. Sci. Nat. Zool., Paris (10)16(14):209-356.

KROMBEIN, K. V.

1957. A generic review of the Amiseginae, a group of phasmatid egg parasites and notes on the Adelphinae. Trans. Amer. Ent. Soc. 82:147-215.

LINSENMAIER, W.

1959. Revision der familie Chrysididae. Mitt. Schweiz. Ent. Ges. 32:1-232.

Perez-D'Angello, V.

1968. Insectos asociados a los nidos de Hypodynerus lachesis (Lepeletier). Rev. Chilena Ent. 6:131-134.

Rứz, F.

1938. Observaciones biologicas de algunos insectos chilenos. Rev. Univ. 23:148-153.

1939. El Odynerus labiatus Hal. y su biología. Rev. Chilena Hist. Nat. 42:97-105. 

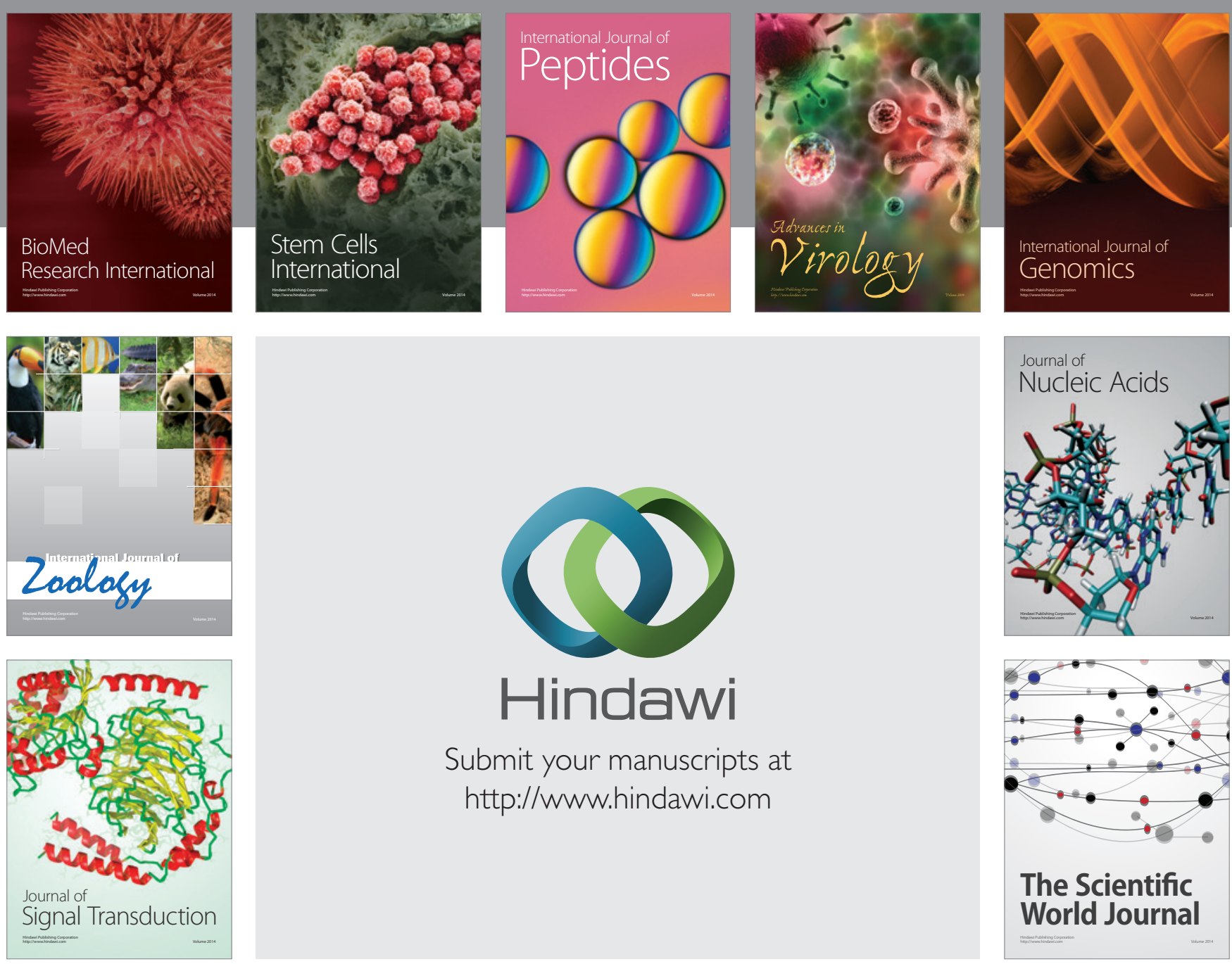

Submit your manuscripts at

http://www.hindawi.com
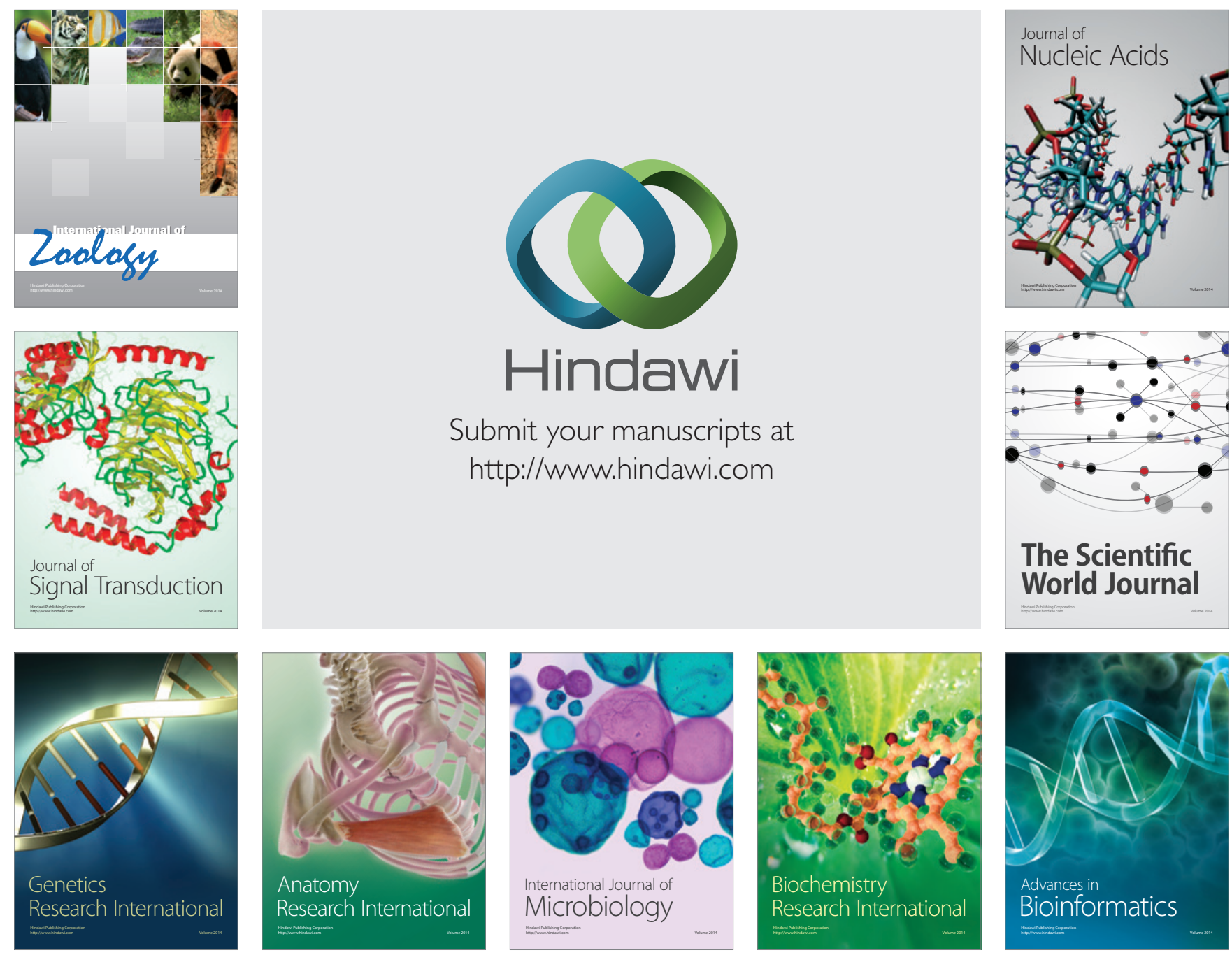

The Scientific World Journal
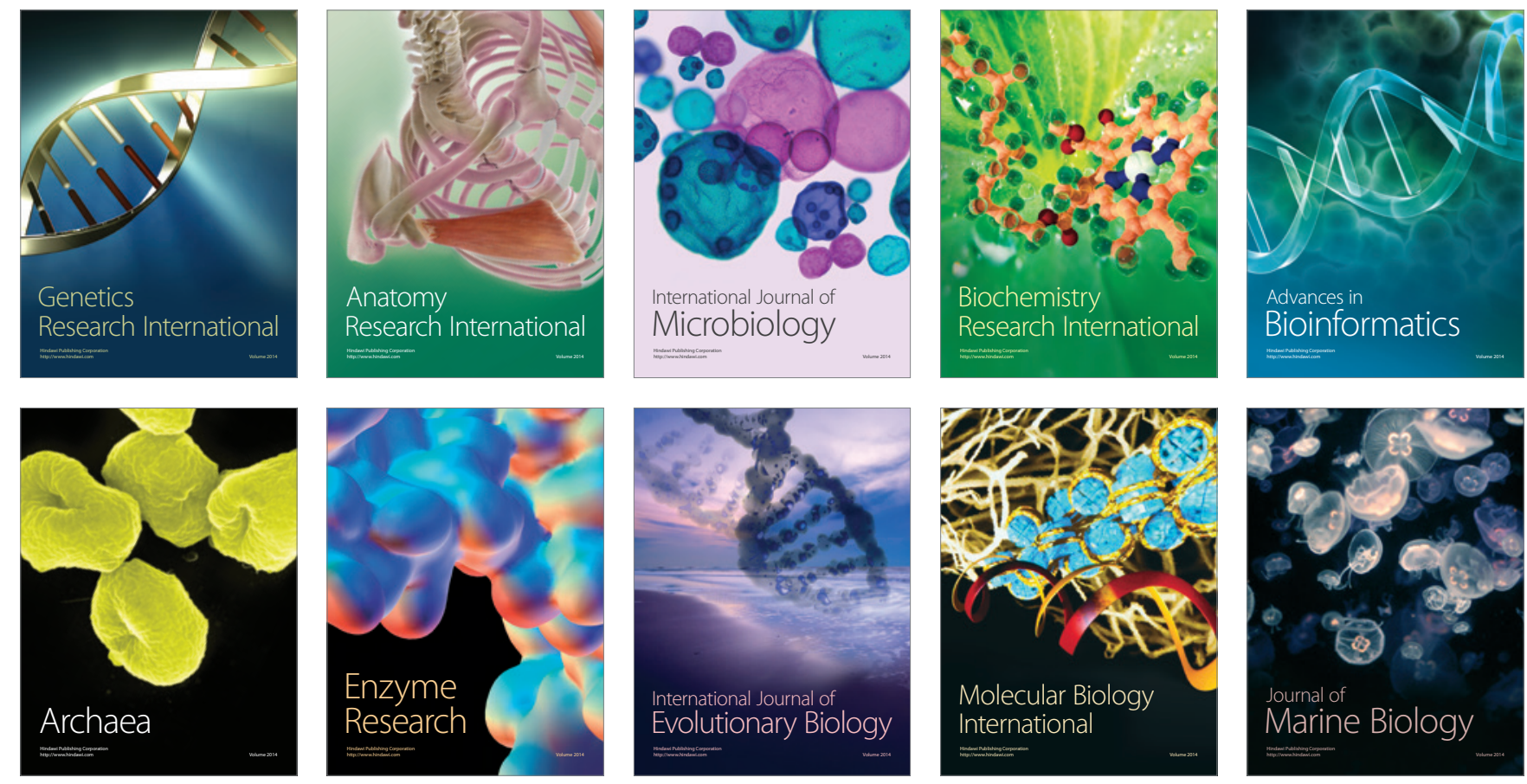